\title{
Plano amostral para cálculo de densidade larvária de Aedes aegypti e Aedes albopictus no Estado de São Paulo, Brasil*
}

\author{
Sampling desing for larval density computation of Aedes Aegypti and Aedes \\ albopictus in the State of S. Paulo, Brazil*
}

\author{
Maria Cecília G.P.Alves"*, Sérgio de M.Gurgel**, Maria do Carmo R.R. de Almeida**
}

\begin{abstract}
ALVES,M.C.G.P. et al. Plano amostral para cálculo de densidade larvária de Aedes aegypti e Aedes albopictus no Estado de São Paulo, Brasil. Rev. Saúde públ., S. Paulo, 25: 251-6, 1991. O Programa de Controle de Vetores de Febre Amarela e Dengue, desenvolvido pela Superintendência de Controle de Endemias do Estado de São Paulo, Brasil, prevê a realização de pesquisa para avaliação da densidade larvária de Aedes aegypti e Aedes albopictus em edificações de municípios com infestação domiciliar. Descreve-se o plano amostral que vem sendo aplicado, desde outubro de 1987, nos municípios da Região de Presidente Prudente. Para acompanhamento da densidade está sendo utilizado o índice de Breteau. São sorteados, nos municípios infestados, mensalmente e de forma independente, amostras de edificações para a obtenção das estimativas do Índice. $O$ plano amostral prevê a seleção de conglomerados em 2 estágios: quadras e edificações. $O$ tamanho da amostra foi definido estimando-se o coeficiente da correlação intraconglomerado e variância relativa por elemento através de pesquisas realizadas anteriormente em municípios do Serviço Regional de São José do Rio Preto. O plano propõe que os valores relativos ao tamanho da amostra sejam atualizados periodicamente em função dos valores obtidos para o estimador do f́ndice de Breteau e sua variância, em meses anteriores.
\end{abstract}

Descritores: Aedes. Larva. Amostragem. Controle de mosquitos.

\section{Introdução}

Índices de densidade larvária têm sido usados em estudos de distribuição e densidade dos vetores da febre amarela e dengue. No Estado de São Paulo adotou-se o Índice de Breteau como indicador dos níveis de infestação domiciliar por Aedes aegypti e Aedes albopictus. Esse índice é referido por $\mathrm{Lok}^{4}$ como sendo o melhor na estimação de densidade larvária. Envolve o dimensionamento dos criadouros existentes, pela contagem dos recipientes com larvas, dando indicação da intensidade da infestação, e não perde de vista a avaliação de sua extensão, uma vez que associa recipientes a casas. Essa associação permite maior aproximação do indicador ao conceito de risco. O seu emprego na avaliação da densidade larvária do Aedes albopictus, que é um vetor de hábitos silvestres, está relacionado com o objetivo de se medir somente a in-

* Trabalho apresentado no $8^{2}$ Simpósio Nacional de Probabilidade e Estatística, Rio de Janeiro, 1988.

** Superintendência de Controle de Endemias (SUCEN) São Paulo, SP - Brasil

Separatas/Reprints: M.C.G.P. Alves - Rua Paula Souza, 166 01027 - São Paulo, SP - Brasil.

Publicação financiada pela FAPESP. Processo Medicina 90/4602-1. festação associada ao ambiente domiciliar.

$O$ conhecimento dos niveis de infestação permite avaliação do risco de aparecimento de surtos e/ou epidemias de dengue e febre amarela. Áreas com índices superiores a 5 são considerados locais onde o contato homem/vetor é significante para o risco de transmissão das doenças. A adoção desse limite de segurança baseia-se em dados sobre a capacidade vetorial do Aedes aegypti em relação a febre amarela. ${ }^{6}$

O Programa de Controle de Febre Amarela e de Dengue, desenvolvido a partir de 1985 no Estado de São Paulo pela Superintendência de Controle de Endemias - SUCEN, objetiva impedir a ocorrência das doenças, evitando a circulação de fontes de infecção e o aparecimento de condições, em relação aos vetores, que propiciem a transmissão5 . A medição do Índice de Breteau deve ser efetuada mensalmente em municípios onde foi constatada a instalação de infestação domiciliar.

O programa propőe, desde a implantação, a utilização de técnicas de amostragem na obtenção dos dados necessários ao cálculo do índice, apoiado na necessidade de obter medidas de maneira ágil, em intervalos mensais. $O$ uso de amostragem probabilística possibilita a estimação do índice de Breteau associada a determinação de intervalos de confiança, dando indicaçōes dos valores limites entre os quais provavelmente se encontra o valor populacional. 
O presente trabalho objetiva descrever o plano amostral que vem sendo aplicado desde outubro de 1987 nos municípios do Serviço Regional(SR) de Presidente Prudente da SUCEN, e ilustrá-lo com resultados obtidos.

\section{Metodologla}

A população objeto é constituída pelas edificaçōes existentes na zona urbana dos municípios, à exceção dos pontos estratégicos (locais propícios à procriação de mosquitos, visitados quinzenalmente), e das construçőes situadas acima do terceiro andar de prédios. Essas edificações (unidades secundárias de amostragem) estão agrupadas em quadras (unidades primárias de amostragem), identificadas e mapeadas pela SUCEN a partir de informações fornecidas pelas prefeituras locais.

\section{Estimador da Densidade Larvária}

A densidade larvária será medida pelo Índice de Breteau (IB), dado pelo número de recipientes com larvas de Aedes aegypti ou Aedes albopictus (recipientes positivos) em 100 edificações pesquisadas, e expresso pela média

$$
\overline{\mathrm{Y}}=\frac{\sum_{i}^{\mathrm{N}} \mathrm{Yi}}{\mathrm{N}}, \text { sendo IB }=100 \overline{\mathrm{Y}}
$$

- onde $\mathbf{Y i}$ é o número de recepientes positivos na edificação i;

$\mathbf{N}$ é o total de edificações.

O estimador é do tipo razão $0^{3}$, uma vez que o processo amostral a ser utilizado é de conglomerados em dois estágios: quadras e edificações, com partilha proporcional ao tamanho da quadra.

$$
\mathrm{r}=\frac{\sum_{\alpha}^{\mathrm{a}} \mathrm{y}_{\alpha}}{\sum_{\alpha}^{\mathrm{a}} \mathrm{x}_{\alpha}}
$$

- onde y $\alpha$ é o número de recipientes positivos encontrados na quadra $\alpha$;

$\mathbf{x} \alpha$ é o número de edificações pesquisadas na quadra $\alpha$;

a é o número de quadras da amostra

Os cálculos de $\overline{\mathrm{Y}}$ e de $\mathrm{r}$ são feitos seguindo os esquemas das Tabelas 1 e 2, construídos com dados de um município hipotético.

Os municípios do Estado de São Paulo estão divididos em dois grupos. Formam o primeiro grupo
Tabela 1. llustração do cálculo do Indice de Breteau na

\begin{tabular}{|c|c|c|c|}
\hline $\begin{array}{l}\text { Quadras } \\
\text { existentes } \\
(\alpha)\end{array}$ & $\begin{array}{l}\text { No de edifica- } \\
\text { ções em cada } \\
\text { quadra }\end{array}$ & $\begin{array}{l}\text { No de recipien- } \\
\text { tes posit. nas } \\
\text { edif. da quadra } \\
\alpha\left(Y_{\alpha}\right)\end{array}$ & $\begin{array}{c}\text { Quadras } \\
\text { selecionadas }\end{array}$ \\
\hline $\begin{array}{l}1 \\
2 \\
3 \\
4\end{array}$ & $\begin{array}{l}22 \\
51 \\
10 \\
65\end{array}$ & $\begin{array}{l}2 \\
1 \\
0\end{array}$ & * \\
\hline \multirow[t]{2}{*}{120} & 34 & $\begin{array}{l}2 \\
0\end{array}$ & * \\
\hline & $N$ & $\sum_{i}^{N} Y_{1}$ & \\
\hline
\end{tabular}
população.

Tabela 2. llustração do cálculo do Indice de Breteau na amostra.

\begin{tabular}{ccc}
\hline $\begin{array}{l}\text { Quadras } \\
\text { selecionadas } \\
(\alpha)\end{array}$ & $\begin{array}{c}N^{2} \text { de edificaçōes } \\
\text { nas quadras sele- } \\
\text { cionadas }\left(x_{\alpha}\right)\end{array}$ & $\begin{array}{c}\text { No de recip. positivo } \\
\text { nas edif. das quadras } \\
\text { selecionadas }\left(y_{\alpha}\right)\end{array}$ \\
\hline 1 & 22 & 2 \\
2 & 10 & 0 \\
3 & 17 & 0 \\
4 & 26 & 1 \\
$\cdot$ & $\cdot$ & $\cdot$ \\
$\cdot$ & $\cdot$ & $\cdot$ \\
$\cdot$ & $\cdot$ & $y_{\alpha}$ \\
$\alpha$ & $x_{\alpha}$ & $\cdot$ \\
$\cdot$ & $\cdot$ &. \\
$\cdot$ & $\cdot$ & 0 \\
20 & 34 & $\sum_{\alpha} y_{\alpha}$ \\
\hline
\end{tabular}

os municípios onde o índice é calculado para a zona urbana como um todo. Nos municípios do segundo grupo, municípios grandes, o índice é calculado para domínios de estudo, nos quais o plano amostral é aplicado separadamente, com a finalidade de obter estimativas com a fixada precisão desejada. A estratificação é utilizada com o objetivo de detalhar a informação sobre infestação e melhor direcionar as medidas de controle. Para cada estrato h o estimador $e^{3}$

$$
\mathrm{r}_{\mathrm{h}}=\frac{\sum_{\alpha}^{a_{h}} \mathrm{y}_{\mathrm{h} \alpha}}{\sum_{\alpha}^{a_{h}} \mathrm{x}_{\mathrm{h} \alpha}}
$$

- onde yha é o número de recipientes positivos na quadra $\alpha$ do estrato $h$. $\mathbf{x h} \alpha$ é o número de edificaçðes na quadra $\alpha$ do estrato $h$.

ah é número de quadras sorteadas no estrato $\mathbf{h}$. 
$O$ indicador para o conjunto dos estratos é expresso por:

$$
\mathrm{r}=\frac{\sum_{\mathrm{h}}^{\mathrm{H}} \frac{\mathrm{Nh}}{\mathrm{nh}} \mathrm{yh}}{\sum_{\mathrm{h}}^{\mathrm{H}} \frac{\mathrm{Nh}}{\mathrm{nh}} \mathrm{xh}}
$$

- onde Nh é o número de edificações existentes no extrato $h$;

nh é o número de edificações selecionadas no estrato $\mathbf{h}$;

H é o número de estratos.

\section{Precisão do Estimador}

A variância de $\mathbf{r}$ é estimada pela expressão ${ }^{3}$

$$
\operatorname{var}(\mathrm{r})=\frac{1-\mathrm{f}}{\left(\Sigma \mathrm{xd}_{\alpha}^{2}\right.} \frac{\mathrm{a}}{\mathrm{a}-1}\left(\Sigma \mathrm{y}_{\alpha}^{2}+\mathrm{r}^{2} \Sigma \mathrm{x}_{\alpha}^{2} 2 \mathrm{r} \Sigma \mathrm{y}_{\alpha} \mathrm{x}_{\alpha}\right)
$$

Para municípios onde são utilizados estratos:

$$
\begin{gathered}
\operatorname{var}(r)=\frac{1}{\left(\sum_{h}^{H} \frac{N_{h}}{n_{h}} x_{h}\right)^{2}}\left[\sum_{h}^{H} w_{h}\left(\sum_{\alpha}^{a_{h}} y_{h \alpha}-\frac{y_{h}^{2}}{a_{h}}\right)+\right. \\
+r_{h}^{2} \sum_{h}^{H} w_{h}\left(\sum_{\alpha}^{a_{h}} x_{h \alpha}^{2}-\frac{x_{h}^{2}}{a_{h}}\right)-2 \underset{h}{H} \sum_{h}\left(\sum_{\alpha}^{a_{h}} x_{h \alpha} y_{h \alpha}+\right. \\
\left.\left.+\frac{x_{h} y_{h}}{a_{h}}\right)\right] \\
\text { onde } w_{h}=\frac{N_{h}^{2}}{n_{h}^{2}}\left(1-\frac{n_{h}}{N_{h}}\right) \frac{a_{h}}{a_{h}-1}
\end{gathered}
$$

\section{Tamanho da Amostra}

Admitindo-se que $\mathbf{r}$ seja normalmente distribuído, o tamanho da amostra é dado por ${ }^{1,3}$

$$
\mathrm{n}_{\mathrm{O}}=\frac{\mathrm{c}_{\mathrm{y}}^{2}}{\mathrm{cv}^{2}(\mathrm{y})} \cdot \operatorname{deff}
$$

$c^{2}$ y é a variância relativa por elemento do número de recipientes positivos e foi estimada em $22.6 \mathrm{em}$ levantamento realizado nos municípios do Serviço Regional de São José do Rio Preto através de:

$$
\begin{aligned}
c_{y}^{2} & =\frac{s_{y}^{2}}{\overline{y^{2}}} \\
\bar{y} & =\frac{\sum_{i}^{n} y_{i}}{n}
\end{aligned}
$$

$\operatorname{cv}^{2}(\bar{y})$, a variância da média $\bar{y}$ é o valor a ser obtido conforme a precisão desejada e o valor espera- do do estimador, sendo dado por:

$$
\mathrm{cv}^{2}(\overline{\mathrm{y}})=\frac{1}{\overline{\mathrm{y}} 2} \frac{\mathrm{d}^{2}}{\mathrm{z}^{2}}
$$

d é a semi-amplitude do intervalo de confiança;

z é o nível de confiança para $(1-\alpha)=0,95$;

deff é o efeito do desenho, e mede o quanto a variância do estimador foi modificada pela utilização de conglomerados no plano amostral. É calculado pela expressão:

$$
\text { deff }=1+p(b-1)
$$

- onde $\mathbf{b}$ é o número de unidades amostrais a serem sorteadas no $2^{\circ}$ estágio.

p é o coeficiente de correlação intraclasse.

O coeficiente de correlação intraclasse ${ }^{2}$ é a medida da homogeneidade entre os elementos dos conglomerados. A estimativa de seu valor, 0.0256 , foi obtida em municípios do Serviço Regional de São José do Rio Preto por

$$
\begin{gathered}
\mathrm{p}=\left[\frac{\operatorname{var}(\mathrm{r})}{\operatorname{var}(\bar{y})}-1\right] \cdot \frac{1}{(\bar{b}-1)} \\
\bar{b}=\frac{n}{a}
\end{gathered}
$$

O valor inicial do tamanho da amostra, no, sofre a correção para população finita, tornando-se

$$
\mathrm{n}=\frac{\mathrm{n}_{\mathrm{O}}}{1+\frac{\mathrm{n}_{\mathrm{O}}-1}{\mathrm{~N}}}
$$

O número de unidades a serem selecionadas, considerando a percentagem de edificações sem resposta $\mathbf{p}_{R}$, será $^{3}$

$$
\mathrm{n}_{1}=\frac{\mathrm{n}}{1-\mathrm{p}_{\mathrm{R}}}
$$

\section{Processo de Seleção}

A fração de amostragem global é dado por ${ }^{3}$

$$
f=\frac{\mathrm{n}}{\mathrm{N}}=\mathrm{a} \frac{\mathrm{N}_{\alpha}}{\mathrm{N}} \cdot \frac{\mathrm{b}}{\left(1-\mathrm{p}_{\mathrm{R}}\right) \mathrm{N}_{\alpha}}
$$

- onde a é o número de quadras a serem selecionadas;

b é o número de edificaçð̃es a serem selecionadas na quadra;

$\mathrm{N} \alpha$ é o número de edificações na quadra $\alpha$; $\mathbf{N}$ é o número total de edificações;

$\mathbf{p}_{\mathbf{R}}$ é a percentagem de edificações sem resposta.

As unidades amostrais de primeiro estágio são quadras selecionadas com probabilidade propor- 
cional ao tamanho, dado pelo número de edificações existentes $\mathrm{N} \alpha$, sendo $\mathrm{N} \alpha \geq \mathrm{b}$. O número de quadras a serem sorteadas com probabilidade $\mathrm{N}_{\propto} / \mathbb{N}$, é determinado por

$$
\mathrm{a}=\frac{\mathrm{n}}{\mathrm{b}}
$$

As unidades amostrais de segundo estágio são as edificaçð̄es, sorteadas sistematicamente nas quadras selecionadas, utilizando-se, em cada quadra, a fração amostral $b / \mathrm{N} \alpha$ (1- pr). A fim de se manter a fração amostral final fixa, o número de edificações a serem pesquisadas é dado por:

$$
\mathrm{b}^{\prime}=\frac{\mathrm{b}}{\left(1-\mathrm{p}_{\mathrm{R}}\right) \mathrm{N}_{\alpha}} \cdot \mathrm{N}_{\alpha}^{\prime}
$$

- onde $N^{\prime} \alpha$ é o número atual de edificações existentes na quadra $\alpha$.

As edificaçōes estão separadas em dois estratos: aquelas nas quais não é possível efetuar a contagem do número de recipientes com larvas (fechadas, vazias ou com recusa na primeira visita) e aquelas nas quais o levantamento é realizado. Os Índices de Breteau do município são estimados admitindo-se que os índices amostrais no estrato de edificações sem pesquisa são iguais aos do estrato com pesquisa realizada ${ }^{3}$. Desta forma, são consideradas no cálculo do estimador $\mathbf{r}$ as edificaçð̃es efetivamente pesquisadas.

\section{Resultados e Comentários}

O plano amostral é utilizado mensalmente, desde novembro de 1987, nos 28 municípios do Serviço Regional de Presidente Prudente que possuem infestação domiciliar pelos vetores de febre amarela e dengue. A área geográfica de atuação dussse Serviço Regional engloba os municípios dos Sisimunas Unificados e Descentralizados de Saúde, SUDS, de Dracena, Presidente Prudente e Presidente Epitácio, num total de 50 municípios.

Para ilustrar a utilização desse plano de amostragem são apresentados os resultados obtidos com a aplicação nos municipios de Presidente Prudente, Presidente Epitácio e Alfredo Marcondes, no periodo de novembro de 1987 a fevereiro de 1988. Esses municípios possuíam, respectivamente, em nov/87, 32.091, 7.286 e 597 edificações, distribuídas em 2.726, 635 e 49 quadras. Em Presidente Prudente havia interesse em se conhecer o Índice de Breteau para as áreas menores do município, em função da necessidade de se obter detalhamento da informação sobre a densidade larvária que melhor direcionasse as medidas de controle. $O$ município foi dividido em 3 domínios de 6.609 ,
20.335 e 11.147 edificações cada, considerando critérios geográficos e níveis supostos de infestação segundo conhecimento de técnicos da área.

Os tamanhos de amostra foram propostos atravês da determinação do número de edificaçð̃es que se desejava visitar em cada quadra, fixado em 8 nesta aplicação, e da consulta às tabelas correspondentes a esse número de edificações (Tabelas $3 \mathrm{e} 4$ ).

A Tabela 3 resume os tamanhos de amostras a serem utilizadas conforme o IB esperado ( $100 \times \mathrm{r}$ ) e a precisão desejada, indicada através da semiamplitude do intervalo de confiança,

$$
(d=1.96 \sqrt{\operatorname{var}(100 \mathrm{r})})
$$

quando o número de edificações a ser pesquisado por quadra é 8.

Uma vez que a escolha do tamanho da amostra se dá em função da capacidade operacional da SUCEN, no momento da pesquisa, a consulta à Tabcla 4 torna-se mais prática por apresentar a precisão a ser obtida para diferentes tamanhos de amostras desejados e IB esperados.

Após a definição do tamanho da amostra, calculou-se o número de quadras a serem visitadas, $\mathrm{n} / 8$, selecionadas com probabilidade proporcional ao tamanho. Nessas quadras foram sorteadas 8 edificações, em média, através de sorteio sistemático.

Tabela 3. Tamanho da amostra em funçāo do IB e da semi-amplitude do intervalo de confiança, para 8 edificaçōes por quadra.

\begin{tabular}{cccccc}
\hline \multirow{2}{*}{ Semi-amplitude } & \multicolumn{5}{c}{ Índice de Breteau } \\
\cline { 2 - 6 } & 1 & 2 & 3 & 4 & \multicolumn{1}{c}{5} \\
\hline 1,0 & 104 & 414 & 930 & 1.653 & 2.583 \\
2,0 & & 104 & 233 & 414 & 646 \\
3,0 & & & 104 & 184 & 287 \\
4,0 & & & & 104 & 162 \\
5,0 & & & & & 104 \\
\hline
\end{tabular}

Tabela 4. Semi-amplitude do intervalo de confiaça em funçáo do tamanho da amostra e do IB com 8 casas sorteadas por quadra.

\begin{tabular}{cccccc}
\hline \multirow{2}{*}{$\begin{array}{c}\text { Tamanho da } \\
\text { amostra }\end{array}$} & \multicolumn{5}{c}{ Valores do índice de Breteau } \\
\cline { 2 - 6 } & $\mathbf{1}$ & $\mathbf{2}$ & $\mathbf{3}$ & $\mathbf{4}$ & $\mathbf{5}$ \\
\hline 100 & 1,0 & 2,0 & 3,0 & 4,1 & 5,1 \\
150 & 0,8 & 1,7 & 2,5 & 3,3 & 4,2 \\
200 & 0,8 & 1,4 & 2,2 & 2,8 & 3,6 \\
250 & 0,7 & 1,3 & 1,9 & 2,6 & 3,2 \\
300 & 0,6 & 1,2 & 1,8 & 2,3 & 2,9 \\
350 & 0,5 & 1,1 & 1,7 & 2,2 & 2,7 \\
400 & 0,5 & 1,0 & 1,5 & 2,0 & 2,5 \\
450 & 0,5 & 0,9 & 1,4 & 1,9 & 2,4 \\
\hline
\end{tabular}

A Tabela 5 apresenta os Índices de Breteau e respectivos intervalos de confiança obtidos com a aplicação do plano em municípios do SR de 
Tabela 5. Esquema de cálculo para obtenção das estimativas do Indice de Breteau em municípios do Serviço Regional de Presidente Prudente - novembro de 1987 a fevereiro de 1988.

\begin{tabular}{|c|c|c|c|c|c|}
\hline Município & Mês & $\begin{array}{c}N^{2} \text { edificaçōes } \\
\text { pesquisadas } \\
\left(\sum_{\alpha}^{\mathrm{a}} \sum_{x \alpha}\right)\end{array}$ & $\begin{array}{c}N^{2} \text { recipientes } \\
\text { positivos } \\
\left(\sum_{\alpha}^{a} y_{\alpha}\right)\end{array}$ & $\begin{array}{c}\text { Índice } \\
\text { de } \\
\text { Breteau } \\
\left(100 \sum y_{\alpha / \Sigma x \alpha}\right)\end{array}$ & $\begin{array}{c}\text { Intervalo de } \\
\text { confiança' } \\
\text { Índice de } \\
\text { Breteau }\end{array}$ \\
\hline Presidente Epitácio & $\begin{array}{l}\text { nov } \\
\text { dez } \\
\text { jan } \\
\text { fev }\end{array}$ & $\begin{array}{l}213 \\
258 \\
233 \\
232\end{array}$ & $\begin{array}{l}22 \\
26 \\
23 \\
29\end{array}$ & $\begin{array}{l}10,3 \\
10,1 \\
10,1 \\
12,5\end{array}$ & $\begin{array}{l}4,1-16,5 \\
5,4-14,8 \\
5,2-15,0 \\
8,0-17,0\end{array}$ \\
\hline Alfredo Marcondes & $\begin{array}{l}\text { jan } \\
\text { lev }\end{array}$ & $\begin{array}{l}115 \\
173\end{array}$ & $\begin{array}{r}9 \\
15\end{array}$ & $\begin{array}{l}7,8 \\
8,7\end{array}$ & $\begin{array}{l}4,5-11,1 \\
3,7-13,7\end{array}$ \\
\hline Pres. Prudente Setor 1 & $\begin{array}{l}\text { nov } \\
\text { dez } \\
\text { jan } \\
\text { fev }\end{array}$ & $\begin{array}{l}186 \\
147 \\
220 \\
248\end{array}$ & $\begin{array}{r}2 \\
8 \\
11 \\
25\end{array}$ & $\begin{array}{r}1,1 \\
5,5 \\
5,0 \\
10,1\end{array}$ & $\begin{array}{l}0,0-2,2 \\
1,1-9,9 \\
2,0-8,0 \\
5,2-15,0\end{array}$ \\
\hline Pres. Prudente Setor 2 & $\begin{array}{l}\text { nov } \\
\text { dez } \\
\text { jan } \\
\text { fev }\end{array}$ & $\begin{array}{l}197 \\
256 \\
225 \\
255\end{array}$ & $\begin{array}{r}8 \\
15 \\
27 \\
74\end{array}$ & $\begin{array}{r}4,1 \\
5,9 \\
12,0 \\
29,0\end{array}$ & $\begin{array}{r}0,4-7,8 \\
1.3-10,5 \\
6,0-18,0 \\
15,2-42,8\end{array}$ \\
\hline Pres. Prudente Setor 3 & $\begin{array}{l}\text { nov } \\
\text { dez } \\
\text { jan } \\
\text { fev }\end{array}$ & $\begin{array}{l}217 \\
218 \\
227 \\
218\end{array}$ & $\begin{array}{r}7 \\
13 \\
18 \\
5\end{array}$ & $\begin{array}{r}3,2 \\
6,0 \\
7,8 \\
21,6\end{array}$ & $\begin{array}{r}0,5-5,9 \\
2,1-9,9 \\
3,4-12,2 \\
10,6-32,6\end{array}$ \\
\hline
\end{tabular}

Tabela 6. Resultados obtidos com a aplicação do plano amostral em município do Serviço Regional de Presidente Prudente - novembro de 87 a fevereiro de 88.

\begin{tabular}{lcccccc}
\hline Municipio & Mês & $\begin{array}{c}\text { Tamanho } \\
\text { proposto }\end{array}$ & $\begin{array}{c}\text { amostra } \\
\text { obtida }\end{array}$ & $\begin{array}{c}\text { Coeficiente } \\
\text { Var. do IB }\end{array}$ & $\begin{array}{c}\text { Efeito } \\
\text { desenho }\end{array}$ & $\begin{array}{c}\text { Coef. correl. } \\
\text { intra classe }\end{array}$ \\
\hline Presidente Epitácio & nov & 200 & 213 & 30,8 & 1,83 & 0,11 \\
& dez & 250 & 258 & 24,1 & 1,09 & 0,01 \\
& jan & 200 & 233 & 24,5 & 1,50 & 0,06 \\
Alfredo Marcondes & fev & 225 & 232 & 18,5 & 1,01 & 0,01 \\
& jan & 100 & 115 & 42,3 & 0,88 & $-0,017$ \\
Pres. Prudente Setor 1 & fev & 150 & 173 & 29,6 & 1,28 & 0,038 \\
& nov & 150 & 186 & 50,2 & 1,04 & 0,004 \\
& dez & 150 & 147 & 40,9 & 1,14 & 0,017 \\
& jan & 200 & 220 & 30,8 & 0,95 & $-0,0002$ \\
Pres. Prudente Setor 2 & fev & 250 & 248 & 24,7 & 0,89 & $-0,012$ \\
& nov & 200 & 197 & 46,5 & 1,01 & $-0,001$ \\
& dez & 250 & 256 & 40,0 & 1,29 & 0,04 \\
& jan & 200 & 225 & 25,5 & 1,14 & 0,02 \\
Pres. Prudente Setor 3 & fev & 250 & 255 & 24,4 & 0,95 & $-0,007$ \\
& nov & 200 & 217 & 42,2 & 1,31 & 0,04 \\
& dez & 200 & 218 & 33,8 & 1,08 & 0,010 \\
& jan & 200 & 227 & 28,1 & 0,80 & $-0,024$ \\
& fev & 200 & 218 & 22,9 & 1,19 & 0,024 \\
\hline
\end{tabular}

Presidente Prudente.

As estimativas do IB variaram entre $1.01 \mathrm{e}$ 21.56, apresentando intervalos de confiança largos nos diversos municípios. Os valores dos coeficientes de variação refletem a limitada precisão dessas estimativas, relacionada com a capacidade operacional da SUCEN para a realização dos levantamentos. Foram pesquisadas, em média, 213 edificações por mês em cada município. Essas amostras, entretanto, apresentaram resultados que acompanhados ao longo do período, forneceram a informação necessária para avaliar a tendência da infestação dos vetores em cada município.

A Tabela 6 apresenta dados que permitem avaliar a eficiência e operacionalidade do plano amostral e serão úteis na proposição de planos futuros.

A adequação do sistema de referência aos propósitos do plano pode ser avaliada pela compa- 
ração entre os tamanhos de amostra esperados e os obtidos efetivamente. Nessa experiência não houve diferença significativa entre esses dois grupos indicando que o mapeamento realizado pela SUCEN está atualizado em relação ao número de edificações.

O grau de homogeneidade dos conglomerados aproximou-se de zero (valor mediano de 0,02), sendo negativo em $28 \%$ das amostras, denotando a existência de grande variabilidade dentro dos conglomerados em relação ao número de recipientes positivos. Indica como satisfatórios o desenho amostral adotado e a utilização de quadra como unidade primária de amostragem.

O valor mediano do efeito do desenho foi 1.09 apontando para a possibilidade de calcular as variâncias das estimativas utilizando fórmulas simplificadas. Essa possibilidade deve ser investigada em estudos futuros.

\section{Agradecimentos}

Agradecemos à equipe do Serviço Regional de Presidente Prudente, pela aplicação do plano nos municípios da região e pela participação na avaliação operacional da experiência.

ALVES, M.C.G.P. et al. [Sampling design for larval density computation of Aedes aegypti and Aedes albopictus in the State of S. Paulo, Brazil] Rev. Saúde públ., S. Paulo, 25: 251-6, 1991. The Yellow Fever and Dengue Vector Control Program developed by the Superintendency for the Control of Endemic Diseases in the State of S. Paulo recommends Aedes aegypti and Aedes albopictus larval density monitoring in cities with domiciliar infestation. The sampling plan which has been applied in the countries of the Presidente Prudente region
(SP- Brazil) since 1987 is described. The infestation is measured by using the Breteau Index. A sample of buildings is drawn, monthly and independently, in the infested cities,in which measurements are to be made. The sample is stratified and the elementary unit selection is made by using two-stage cluster sampling: of blocks and buildings. The sample sizes were defined using the coefficient of variation and the intraclass correlation as estimated for towns covered by the Regional Health Service of S. José do Rio Preto. These sizes must be corrected periodically in accordance with the values of the Breteau Index and its variance obtained in previous months.

Keywords: Aedes. Larva. Sampling studies. Mosquito control.

\section{Referências Bibliográficas}

1. COCHRAN, W. G. Sampling techniques. New York, John Wiley \& Sons, 1977.

2. HANSEN, M. H. et al. Sample survey methods and theory. New York, John Wiley \& Sons, 1953.

3. KISH, L. Survey sampling. New York, John Wiley \& Sons, 1965.

4. LOK, C. K Singapore's dengue haemorragic fever control programe: a case study on the successful control of Aedes aegypti and Aedes albopictus using mainly enviromental measures as a port of integrated vector control. Singapore, Ministry of Health of Singapore, 1985.

5. SUPERINTENDENCIA DE CONTROLE DE ENDEMIAS (SUCEN). Programa de controle de Aedes aegypti e Aedes albopictus no Estado de São Paulo. São Paulo, 1985 [Mimeografado].

6. WORLD HEALTH ORGANIZATION. A system of world-wide surveillance for vectors. Whly epidem. Rec., 47: 73-84, 1972.

Recebido para publicaçāo em 30111/11989 Reapresentado em 07/06/1991 Aprovado para publicação em 18/06/1991 